\title{
Comparación leche de vaca y sustituto lácteo en la alimentación de terneros mestizos destetados precozmente
}

\author{
Comparison of cow's milk and milk substitute in the feeding of early-weaned crossbred calves
}

José Humberto Vera Rodríguez ${ }^{1}$

\section{Resumen}

En la finca Santa Bárbara de la parroquia Canuto-Chone-Manabí-Ecuador se evaluaron dos sistemas de alimentación láctea y dos períodos de destete: $\mathrm{T}_{1}$ ) Leche de vaca, destete 30 días; T2) Leche de vaca, destete 45 días; T3) Sustituto lácteo comercial Ecomilk E.Q., destete 30 días; $\mathrm{T}_{4}$ ) Sustituto lácteo comercial Ecomilk E.Q., destete 45 días, sobre los parámetros productivos: Peso $(\mathrm{Kg})$, consumo de alimento $(\mathrm{Kg})$, ganancia de peso $(\mathrm{Kg})$, conversión alimenticia $(\mathrm{Kg} / \mathrm{Kg})$ y análisis de costos $(\mathrm{U} \$)$ en 20 terneros mestizos $75 \%$ Sahiwal / 25\% Jersey a partir de 1 día de nacido con un peso promedio de $34.70 \pm 0.75 \mathrm{Kg}$; Los terneros fueron estabulados individualmente y alimentados con heno de alfalfa, concentrado pre-inicial con $25 \%$ de proteína y agua a voluntad durante 90 días de estudio. El análisis estadístico de las variables estudiadas se realizó desde un diseño completamente aleatorizado factorial (2x2) con 5 repeticiones por tratamiento, analizados en el Paquete Estadístico IBM SPSS versión 20. No existiendo diferencias significativas entre los factores en estudio y su interacción sobre los parámetros productivos; mientras que el análisis de costos mostró beneficio del destete precoz a los 30 días, con ventajas al utilizar el sustituto lácteo. Concluyendo que, a pesar de no presentarse diferencias significativas entre los tratamientos y variables en estudio, el análisis de costos por Kg de alimento consumido en base a materia seca, mostró beneficios a favor del destete precoz 30 días, al combinar este factor con sustituto lácteo, representando así un ahorro del 15\%.

Palabras clave: Destete precoz; leche: sustituto lácteo; ternero.

\section{Abstract:}

In the Santa Bárbara farm from Canuto-Chone-Manabí-Ecuador parish, two dairy feeding systems and two weaning periods were evaluated: $\mathrm{T}_{1}$ ) Cow's milk, weaning 30 days; T2) Cow's milk, weaning 45 days; T3) Ecomilk E.Q. Commercial Dairy Substitute, weaning 30 days; $\mathrm{T}_{4}$ ) Ecomilk EQ commercial dairy substitute, weaning 45 days, on the productive parameters: Weight $(\mathrm{Kg})$, feed consumption $(\mathrm{Kg})$, weight gain $(\mathrm{Kg})$, feed

\footnotetext{
1 Magíster en Zootecnia. Docente Investigador, Instituto Superior Tecnológico Enrique Noboa Arízaga, La Troncal - Ecuador. email: humbertorichi@hotmail.com; https://orcid.org/0000-0003-3027-059X
}

Recibido: 19/07/2020 - Aprobado: 21/09/2020 
conversion $(\mathrm{Kg} / \mathrm{Kg})$ and cost analysis $(\mathrm{U} \$)$ in 20 crossbred calves 75\% Sahiwal / 25\% Jersey from 1 day of birth with an average weight of $34.70 \pm 0.75 \mathrm{Kg}$; The calves were housed individually and fed with alfalfa hay, pre-initial concentrate with $25 \%$ protein and water ad libitum for 90 days of study. The statistical analysis of the variables studied was carried out from a completely randomized factorial design (2x2) with 5 repetitions per treatment, analyzed in the IBM SPSS Statistical Package version 20. There were no significant differences between the factors under study and their interaction on the productive parameters; while the cost analysis showed a benefit of early weaning at 30 days, with advantages when using the milk substitute. Concluding that, despite not presenting significant differences between the treatments and variables under study, the analysis of costs per feed kilogram consumed based on dry matter, showed benefits in favor of early weaning 30 days, when combining this factor with milk substitute, thus representing a saving of $15 \%$.

Keywords: Early weaning; milk: milk substitute; calf.

\section{Introducción}

Los costos de producción durante la crianza del ternero son muy altos por lo que los ganaderos deben de optar por sistemas alternativos de crianza económicos, pero de buena calidad que permita su buen desarrollo, permitiendo destetarlos a una edad temprana.

Para lograr una ganadería productiva y estable, se precisa de buenos resultados en la cría de terneros y su reemplazo, pues las pérdidas que ocurren durante este proceso, debido a las bajas tasas de ganancia de peso vivo no se recuperan. La garantía del crecimiento y desarrollo adecuado del ternero depende de numerosos factores que, tienen que ver con la alimentación y el manejo (Ybalmea, R., 2015).

Varios programas de alimentación para terneros sirven como alternativas de crianzas dentro de hatos ganaderos capaces de mejorar la producción (Pavan, M., 2018). El uso de alimentos lácteos como el calostro, yogurt, leche fresca y reemplazantes lecheros en sistemas de cría artificial y amamantamiento restringido constituyen las principales temáticas. También se comentan algunos resultados obtenidos en la elaboración y utilización de piensos de inicio y raciones integrales, el uso de pastos y forrajes y la suplementación mineral a animales jóvenes. Se tratan, además, asuntos relacionados con la conducta y el bienestar animal en terneros jóvenes, como herramienta para reducir la susceptibilidad a las enfermedades y mejorar el comportamiento productivo (Ybalmea, R., 2015).

El sustituto lácteo es el reemplazante de la leche en el mercado, los hay con una variada calidad nutricional, un excelente sustituto es el que se parece más a la leche, siendo el objetivo principal de garantizar el cierre de la gotera esofágica y para lograr 
esto se deben tener en cuenta algunos factores que estimulan el funcionamiento de esta y hagan que el sustituto lácteo vaya directo al abomaso donde se forma el correcto coágulo y la formación de paracaseinato de calcio (Pavan, M., 2018).

El destete precoz es la supresión temprana del alimento lácteo del ternero, con la aplicación de esta práctica, los ganaderos pueden ganar adicionalmente dinero por cabeza de ganado por año, además bajo este régimen las vacas continuarán produciendo un becerro cada 12 o 13 meses. Por lo que resulta ser una alternativa viable para incrementar la productividad, rentabilidad de los ranchos y la utilización en forma eficiente de los recursos naturales en los predios, así como su conservación a largo plazo. Por otro lado, permite mantener la producción de becerros en forma sostenida durante la proyección y con ello diluir el efecto de los costos fijos en los flujos financieros, haciendo más eficiente económicamente la utilización de los recursos naturales (Medina, S. M, et al., 2017).

Dentro de las opciones para disminuir los costos de crianza de terneros está en reducir el tiempo de destete, fortaleciendo el sistema de crianza durante la fase de lactancia con alimentos de calidad que estimulen su buen desarrollo. Bajo este contexto, en la Finca Santa Bárbara de la Parroquia Canuto del Cantón Chone, se realizó la comparación de leche de vaca y sustituto lácteo en la alimentación de terneros mestizos destetados precozmente para medir su comportamiento productivo y económico.

\section{Revisión de literatura}

Las características anatómicas y fisiológicas de los terneros le confieren una adaptabilidad y una elevada potencialidad de su sistema digestivo que le permite el tránsito de un alimento como es la leche entera por otro como es el sustituto lechero y obtener buenos resultados. Una de las ventajas más notables en la crianza artificial del ternero es la posibilidad de utilizar sustitutos lecheros, cuyos precios son inferiores a la leche entera, se valora como con el uso de sustituto lechero, es posible ahorrar 180 litros de leche fresca por ternero, comparado con la utilización de leche fresca, con un $\mathrm{Kg}$ de sustituto lechero, se pueden sustituir 5,81 litros de leche, ya que reduce el costo de la crianza y destinar aproximadamente el $50 \%$ de la leche que consume el ternero, para el consumo humano (Quintero, B. G., 2007).

El empleo de sustitutos lácteos no afecta la conducta de los terneros presentando indicadores financieros rentables, pudiendo utilizar hasta el destete, siempre y cuando se estimule la rumia con la presencia de fibra (Vazquez, Á. C. A., et al., 2017).

El destete precoz es una alternativa tecnológica eficiente y eficaz para mejorar la productividad del rodeo de cría y que existen diferentes formas de realizarlo, que son adaptables al sistema de producción ganadero que se trate. El manejo nutricional del ternero destetado precozmente y manejado en confinamiento se plantea como una 
estrategia que permite obtener ganancias superiores a las que se logran en pastoreo, con conversión del alimento buenas (Simeone, A., y Beretta, V., 2016). Hay que tomar muy en cuenta la suplementación de terneros ya que mejora de manera importante su ganancia diaria de peso (Pineda, V., y Valiente, O. L., 2019).

\section{Materiales y métodos}

El trabajo experimental se llevó a cabo en la Región Tropical del Ecuador en la finca "Santa Bárbara", ubicada en la Parroquia Canuto del Cantón Chone-Manabí-Ecuador, geográficamente ubicada en las coordenadas o47’54,05” S - 8007’39,06” O con una elevación de 26 m.s.n.m., con una topografía irregular. Presenta una temperatura media anual de $25^{\circ} \mathrm{C}$., presenta una precipitación promedio anual de $1759 \mathrm{~mm}$ y su heliofanía es de 677 horas sol/año, velocidad del viento 3.5 m/s (INAMHI, 2013).

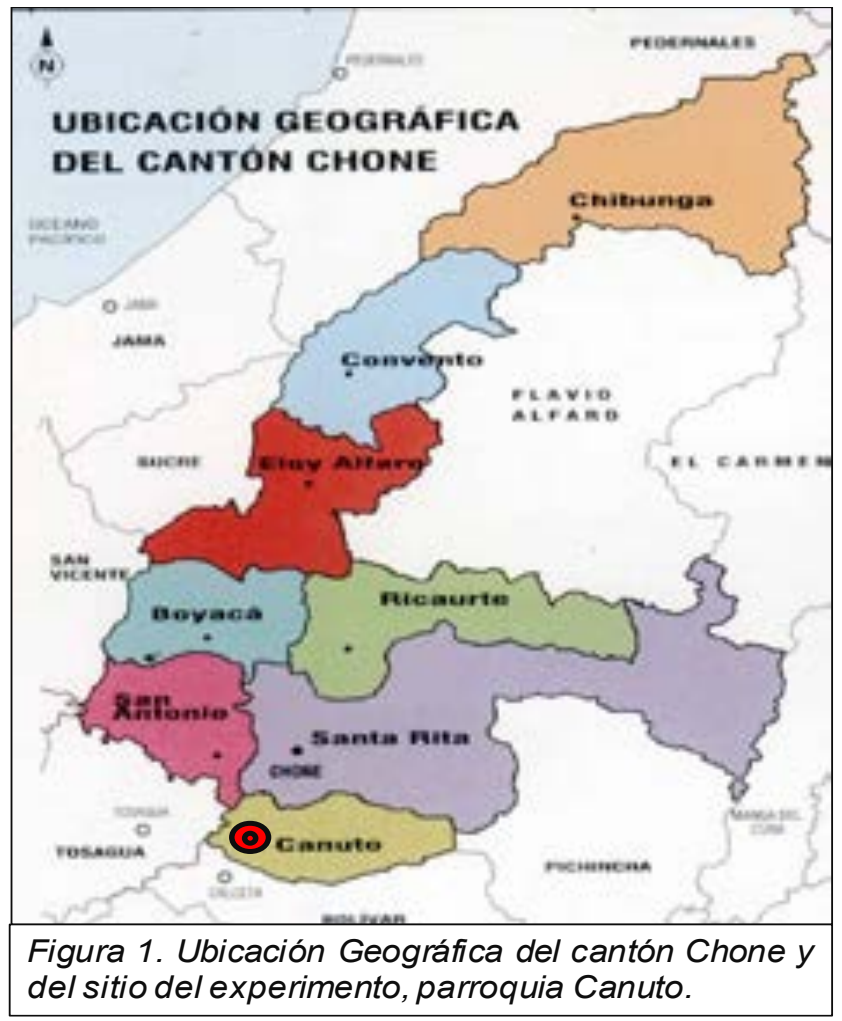

Figura 1: Ubicación geográfica del cantón Chone y del sitio del experimento Parroquia Canuto.

El trabajo experimental tuvo una duración de 90 días, se utilizaron 20 terneros mestizos con un cruce racial 75\% Sahiwal / 25\% Jersey desde el primer día de nacido, habiendo calostrado durante 24 horas con su respectiva madre, posteriormente fueron 
alojados en jaulas individuales (Unidad Experimental) de $1.92 \mathrm{mts} 2(1.20 \mathrm{~m} \times 1.60 \mathrm{~m})$, como cama se usó tamo de arroz.

Se evaluaron dos sistemas de alimentación láctea y dos periodos de destete, asignados sus tratamientos de la siguiente manera: $\mathrm{T} 1$ ) leche de vaca, destete 30 días; $\mathrm{T}_{2}$ ) leche de vaca, destete 45 días; $\mathrm{T}_{3}$ ) sustituto lácteo comercial Ecomilk E.Q., destete 30 días; $\mathrm{T}_{4}$ ) sustituto lácteo comercial Ecomilk E.Q., ver Tabla 1, destete 45 días. El suministro de la alimentación láctea se proporcionó a una temperatura de $37^{\circ} \mathrm{C}$ mediante cubetas, repartidos en dos tomas diarias o6hoo y 17 hoo, proporcionando 2 litros por horario según correspondiera el tratamiento. Complementariamente fueron alimentados los terneros con heno de alfalfa, concentrado pre-inicial con $25 \%$ de proteína (ver Tabla 2) y agua a voluntad.

Tabla 1: Composición Sustituto Lácteo comercial Ecomilk E.Q.

\begin{tabular}{ll}
\hline \multicolumn{1}{c}{ Composición } & \multicolumn{1}{c}{ Unidad } \\
\hline Humedad & $4,5 \%$ \\
Proteína bruta & $22,0 \%$ \\
Materia grasa bruta & $17,0 \%$ \\
Ceniza bruta & $7,0 \%$ \\
Fibra bruta & $0,6 \%$ \\
Vitamina A & $25.000 \mathrm{UI}$ \\
Vitamina D3 & $10.000 \mathrm{UI}$ \\
Vitamina E (acetato alfa tocoferol) & $100 \mathrm{mg}$ \\
Vitamina C & $250 \mathrm{mg}$ \\
Hierro (sulfato ferroso monohidratado) & $51 \mathrm{mg}$ \\
\hline
\end{tabular}

Tabla 2: Composición Alimento Balanceado Pre Inicial

\begin{tabular}{ll}
\hline \multicolumn{1}{c}{ Composición } & \multicolumn{1}{c}{ Unidad } \\
\hline Proteína cruda (mín.) & $25.0 \%$ \\
Grasa cruda (mín.) & $5.0 \%$ \\
Fibra cruda (máx.) & $8.0 \%$ \\
Ceniza (máx.) & $8.0 \%$ \\
Calcio (min.) & $1.0 \%$ \\
Fósforo (min.) & $0.5 \%$ \\
\hline
\end{tabular}


Las variables objeto de análisis en los parámetros productivos fueron: Peso inicial (Kg); Peso final (Kg); Consumo de alimento en base a materia seca MS (Kg) durante los periodos: 30 días, 30 a 45 días, 45 a 90 días y todo el período hasta los 90 días de edad; Ganancia diaria de peso ( $\mathrm{Kg} /$ día) durante los períodos: 30 días, 30 a 45 días, 45 a 90 días y todo el período hasta los 90 días de edad; Conversión alimenticia $(\mathrm{Kg} /$ $\mathrm{Kg}$ ) durante los periodos: 30 días, 30 a 45 días, 45 a 90 días y todo el periodo hasta los 90 días de edad y análisis de costos (U\$) durante los 30 días, 30 a 45 días, 45 a 90 días y todo el período hasta los 90 días de edad. Para el pesaje de los alimentos y los terneros se utilizó una balanza digital marca JONTEX CE Modelo oo8 con capacidad hasta $300 \mathrm{~kg}$ con una precisión de 0,001-0,2.

El análisis estadístico de las variables estudiadas se realizó con un diseño completamente aleatorizado DCA factorial ( $2 \times 2$ ) con 5 repeticiones por tratamiento, para las comparaciones entre las medias se aplicó en los casos requeridos la Prueba de Tukey con ( $\mathrm{p}<0.05)$, analizados en el Paquete Estadístico IBM SPSS versión 20.

\section{Resultados y discusión}

A continuación, se detallan los resultados del análisis estadístico para cada una de las variables estudiadas.

Peso inicial y final (Kg). El peso inicial de los animales que fueron utilizados en el experimento, fue bastante uniforme, tal como se aprecia en la tabla 3; no se encontró diferencias significativas entre los grupos que se conformaron, por lo que se considera un indicativo adecuado al modelo de análisis utilizado que consistió en una distribución Totalmente al Azar. Por otra parte, el peso final tampoco mostró diferencias significativas entre los tratamientos, indicativo de un comportamiento semejante durante el desarrollo de todo el período experimental.

Tabla 3: Peso Inicial y Final de los terneros (Kg):

\begin{tabular}{lllllll}
\hline \multirow{2}{*}{ Variables } & \multicolumn{2}{c}{ Leche entera } & \multicolumn{2}{c}{ Sustituto lechero } & \multirow{2}{*}{ Sig. } & Error típico \\
\cline { 2 - 5 } & Destete 30 días & Destete 45 días & Destete 30 días & Destete 45 días & & \\
\hline Peso inicial & $35,30 a$ & $34,00 a$ & $34,70 a$ & $34,80 a$ & NS & 2,65 \\
Peso final & $98,86 a$ & $100,78 a$ & $107,14 a$ & $99,44 a$ & NS & 3,77 \\
\hline
\end{tabular}

a, b y c: letras distintas en una misma hilera indican diferencias estadísticas según Tukey (0,05). NS: No significativo

El peso de los terneros al nacimiento puede oscilar entre 30 y $45 \mathrm{~kg}$ sin diferenciación de sexo (Lagger, J., 2010). Los terneros que participaron en la investigación presentaron un peso inicial comprendido en el intervalo normal de referencia para su cruce racial, ver tabla 3. 
Consumo de alimento en base a materia seca M. S. (Kg/día). El Consumo diario de MS para los diferentes tratamientos comparados durante los tres períodos en los que se dividió el estudio y el acumulado promedio desde el inicio hasta los 90 días se muestra en la tabla 4.

El análisis de varianza, arrojó que no existieron diferencias entre los alimentos lácteos utilizados, ni entre los momentos en los que se produjo el destete, sin interacción significativa entre ambos factores; por lo tanto, entre los tratamientos combinados tampoco se encontró diferencias significativas, en cada uno de los períodos analizados.

Tabla 4: Comportamiento del Consumo de alimento en base a materia seca M. S. (Kg/día).

\begin{tabular}{|l|l|l|l|l|l|l|}
\hline \multirow{2}{*}{ Períodos } & \multicolumn{2}{|c|}{ Leche entera } & \multicolumn{2}{c|}{ Sustituto lechero } & \multirow{2}{*}{ Sig. } & \multirow{2}{*}{ Error típico } \\
\cline { 2 - 5 } & \multicolumn{1}{|c|}{ Destete 30 días } & Destete 45 días & Destete 30 días & \multicolumn{1}{|c|}{ Destete 45 días } & & \\
\hline Primeros 30 días & $0,85 a$ & $0,93 a$ & $0,94 a$ & $0,85 a$ & NS & 0,06 \\
\hline De 30 a 45 días & $2,35 a$ & $2,16 a$ & $2,34 a$ & $2,15 a$ & NS & 0,23 \\
\hline De 45 a 90 días & $2,17 a$ & $2,31 a$ & $2,26 a$ & $2,22 a$ & NS & 0,08 \\
\hline Todo el período & $1,76 a$ & $1,83 a$ & $1,84 a$ & $1,76 a$ & NS & 0,08 \\
\hline
\end{tabular}

a, b y c: letras distintas en una misma hilera indican diferencias estadísticas según Tukey (0,05). NS: No significativo

Lagger, J. (2010) sostiene que, el crecimiento de los tejidos magros va a depender de la cantidad y calidad de proteína de los componentes en la alimentación, mismas que deben ser equilibrados nutricionalmente, así se lograrían animales más saludables. Juliano, N., et al., (2016) en su investigación, probó diferentes composiciones químicas de dietas líquidas como sustituto lácteo, no encontrando diferencias significativas en el tiempo al destete, ganancia diaria de peso vivo, estimación de consumo de materia seca en terneros machos de raza Holando-Argentino.

Ganancia diaria (Kg/día). El análisis de los resultados en relación a la ganancia diaria, se presenta en la tabla 5, el mismo mostró que para esta variable en los diferentes períodos no se encontró interacción significativa entre los dos factores en estudio, por lo que se analizaron los resultados de las combinaciones correspondientes entre los niveles de dichos factores; encontrándose en este caso que tampoco hubo diferencias significativas para los mismos.

Tabla 5: Comportamiento de la Ganancia diaria (Kg/día).

\begin{tabular}{|l|l|l|l|l|l|l|}
\hline \multirow{2}{*}{ Períodos } & \multicolumn{2}{|c|}{ Leche entera } & \multicolumn{2}{c|}{ Sustituto lechero } & \multirow{2}{*}{ Sig. } & \multirow{2}{*}{ Error típico } \\
\cline { 2 - 5 } & \multicolumn{1}{|c|}{ Destete 30 días } & \multicolumn{1}{|c|}{ Destete 45 días } & \multicolumn{1}{|c|}{ Destete 30 días } & Destete 45 días & & \\
\hline Primeros 30 días & $0,60 a$ & $0,52 a$ & $0,67 a$ & $0,54 a$ & NS & 0,10 \\
\hline De 30 a 45 días & $0,88 a$ & $0,98 a$ & $1,03 a$ & $0,95 a$ & NS & 0,07 \\
\hline De 45 a 90 días & $0,72 a$ & $0,81 a$ & $0,82 a$ & $0,76 a$ & NS & 0,07 \\
\hline Todo el período & $0,71 a$ & $0,74 a$ & $0,80 a$ & $0,72 a$ & NS & 0,05 \\
\hline
\end{tabular}




\section{a, b y c: letras distintas en una misma hilera indican diferencias es- tadísticas según Tukey $(0,05)$. NS: No significativo}

Concordado con lo informado por Cardenas, J. et al. (2015), los terneros que son suplementados presentan ganancias diarias de peso promedio mayor con respecto al grupo control. Por tanto, el uso del concentrado comercial pre-inicial con el $25 \%$ de proteína en la investigación beneficia a los terneros en la ganancia de peso.

Los animales $\mathrm{F} 1$ dan una mayor ganancia diaria de peso en relación a animales de un solo linaje, permitiendo mejorar el peso al destete (Holgado, F. D., et al., 2016), este dato es un indicativo para el tipo de ganado utilizado en la investigación. Cuando el $76 \%$ de los terneros llegan a consumir $>1 \mathrm{Kg}$ de materia seca como iniciador al día al momento del destete, asegura su crecimiento durante el post-destete (Pared, S., 2017), esta información argumenta los resultados obtenidos en la investigación en la tabla 5.

Conversión alimenticia (Consumo diario MS/ Ganancia diaria). La conversión de alimentos, calculada a partir del consumo de MS y la ganancia diarios se refleja en la tabla 6, para cada uno de los períodos estudiados y para todo el período experimental. En este caso tampoco resultó significativa la comparación entre los factores en estudio y su interacción. Los resultados de la tabla muestran que para las combinaciones (Tratamientos) tampoco se encontraron diferencias significativas en ninguno de los períodos.

Tabla 6: Efecto de la Conversión (Consumo diario MS/ Ganancia diaria).

\begin{tabular}{|l|l|l|l|l|l|l|}
\hline \multirow{2}{*}{ Períodos } & \multicolumn{2}{|c|}{ Leche entera } & \multicolumn{2}{c|}{ Sustituto lechero } & \multirow{2}{*}{ Sig. } & \multirow{2}{*}{ Error típico } \\
\cline { 2 - 5 } & \multicolumn{1}{|c|}{ Destete 30 días } & Destete 45 días & \multicolumn{1}{c|}{ Destete 30 días } & Destete 45 días & & \\
\hline Primeros 30 días & $1,47 a$ & $1,81 a$ & $1,43 a$ & $1,87 a$ & NS & 0,32 \\
\hline De 30 a 45 días & $2,65 a$ & $2,21 a$ & $2,29 a$ & $2,29 a$ & NS & 0,26 \\
\hline De 45 a 90 días & $3,04 a$ & $2,92 a$ & $2,80 a$ & $2,93 a$ & NS & 0,18 \\
\hline Todo el período & $2,50 a$ & $2,47 a$ & $2,29 a$ & $2,46 a$ & NS & 0,18 \\
\hline
\end{tabular}

a, b y c: letras distintas en una misma hilera indican diferencias estadísticas según Tukey (0,05). NS: No significativo

El consumo de alimento es un eje importante en el desarrollo de los terneros y como indica Castro-Flores, P., et al., (2012) un adecuado desarrollo ruminal en animales de reemplazo, tiene que ver más con la alimentación que con la edad de los mismos y esto debe ser considerado al momento del destete para poder desarrollar así animales con una baja incidencia de enfermedades, adecuadas ganancias de peso y que puedan llegar a producir eficientemente.

Análisis de Costos de los resultados. El análisis de los costos se valoró de igual manera, tomando en consideración su variabilidad determinada por el consumo de los diversos alimentos vinculados a cada uno de los tratamientos. En la tabla 7; 
aparecen los resultados del costo para cada uno de los períodos estudiados y para todo el período experimental.

Durante los primeros 30 días del estudio, el costo fue determinado por el tipo de alimentación láctea, encontrándose los costos más altos cuando los animales fueron alimentados con leche de vaca entera, en comparación con aquellos donde se utilizó el sustituto lácteo. En el período de 30 a 45 días por su parte, la determinación del costo estuvo definida fundamentalmente por el momento del destete; encontrándose los valores más altos cuando el consumo lácteo se extendió hasta 45 días, aunque mucho más económico en ese caso cuando se utilizó el sustituto lechero. En el último período del experimento (De 45 a 90 días); los costos de la alimentación fueron relativamente semejantes para todos los tratamientos estudiados.

El resultado de los costos totales durante todo el período (9o días) mostró el beneficio del destete precoz a los 30 días, con ventajas también a favor de la utilización del sustituto lechero, representando así un ahorro del 15\% en los costos por alimentación durante 90 días de estudio por ternero, habiendo sido comparado con el tratamiento más alto que fue a base de leche de vaca y destete a los 45 días.

Tabla 7: Costo total (U\$) de alimento consumido por períodos.

\begin{tabular}{|l|l|l|l|l|}
\hline \multirow{2}{*}{ Variables } & \multicolumn{2}{c|}{ Leche entera } & \multicolumn{2}{c|}{ Sustituto lechero } \\
\cline { 2 - 5 } & \multicolumn{1}{|c|}{ Destete 30 días } & Destete 45 días & \multicolumn{1}{c|}{ Destete 30 días } & \multicolumn{1}{c|}{ Destete 45 días } \\
\hline Hasta los 30 días & 57,42 & 60,14 & 50,77 & 50,10 \\
\hline De 30 a 45 días & 25,83 & 38,46 & 28,55 & 34,73 \\
\hline De 45 a 90 días & 56,03 & 59,18 & 58,33 & 57,24 \\
\hline Todo el período & 139,27 & 157,77 & 137,64 & 142,07 \\
\hline
\end{tabular}

Otra visión de la eficiencia económica lograda con los diferentes tratamientos se muestra en la Figura $\mathrm{N}^{\circ}$ 2, donde aparece el costo promedio por kilogramo de alimento en base a materia seca en cada uno de los períodos y durante los 90 días totales del experimento. En la misma se demuestra que en todos los casos la eficiencia económica alcanzada favorece en todas las etapas a la combinación del destete precoz (3o días) y el sustituto lechero. 


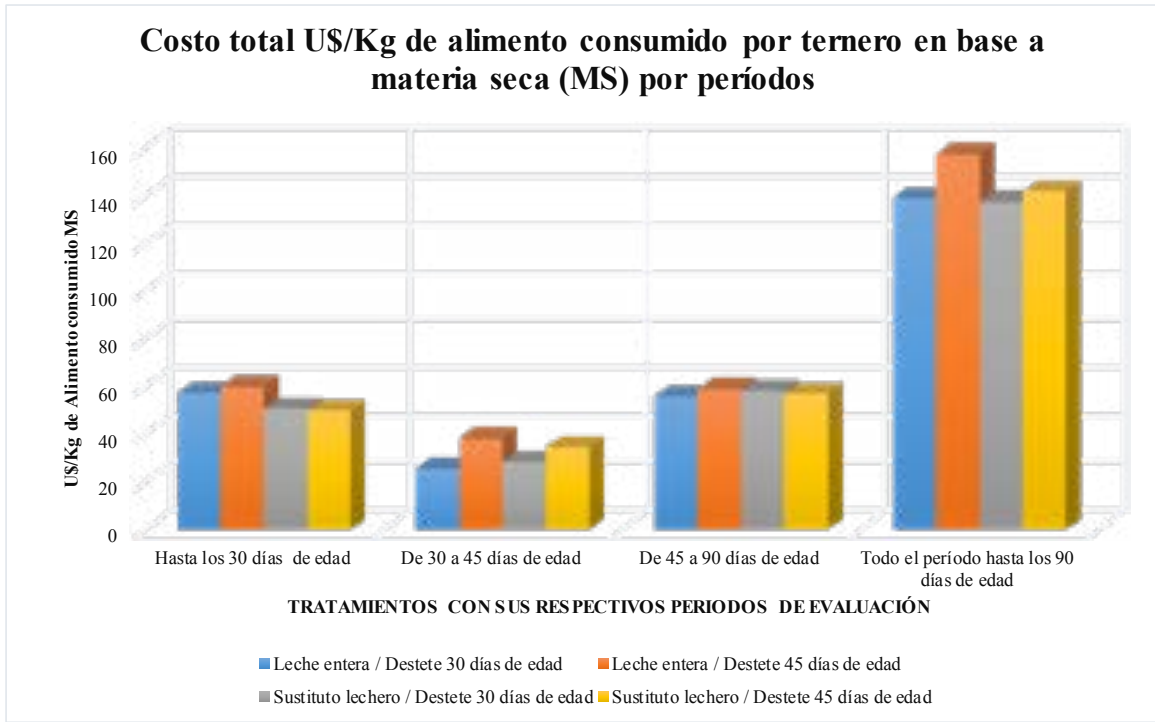

Figura 2: Eficiencia económica de los tratamientos por períodos

Las prácticas del destete precoz muestran que, no obstante, a los costos que implican su implementación, resultan ser alternativas tanto técnicas como económicamente viables para incrementar la productividad y rentabilidad de los ranchos (Moreno, S., et al., 2018).

\section{Conclusiones}

En conclusión, las variables productivas estudiadas mostraron que no existieron diferencias significativas entre los tratamientos estudiados, ni interacción entre los factores que los componen (Tipo de alimento y momento del destete); sin embargo, el análisis de los resultados económicos en función de los costos y la eficiencia económica a partir del costo por kg de alimento, mostró en todos los casos beneficios a favor del destete precoz (3o días) y sobre todo al combinar este factor con el uso del sustituto lechero, representando así un ahorro del $15 \%$ en los costos por alimentación durante 90 días de estudio por ternero, habiendo sido comparado con el tratamiento más alto que fue a base de leche de vaca y destete a los 45 días.

\section{Recomendaciones}

Para futuras investigaciones, se recomienda replicar esta evaluación en animales de genética destinada a la producción cárnica, de seguro se obtendrán excelentes resultados. 


\section{Agradecimiento}

Esta publicación obtuvo el financiamiento de: El Fondo de Asistencia Internacional de los Estudiantes y Académicos Noruegos (SAIH).

\section{Lista de referencias}

Cardenas, J., Maza, A., Cardona, J. (2015). Comportamiento productivo de terneros lactantes suplementados con maíz más torta de algodón en el departamento de Córdoba, Colombia. Revista Colombiana de Ciencia Animal-RECIA, 7(2), 171-178. https://doi.org/10.24188/recia.v7.n2.2015.261

Castro-Flores, P., Elizondo-Salazar, J. A. (2012). Crecimiento y desarrollo ruminal en terneros alimentados con iniciador sometido a diferentes procesos. Agronomía Mesoamericana, 23(2), 343-352. https://revistas.ucr.ac.cr/index.php/agromeso/ article/view/6534

Holgado, F. D., Ortega, M. F., Fernández, J. L., Hernández, M. E. (2016). Ganancia diaria de peso predestete en terneros de vacas criollas y $\mathrm{F}_{1}$ Criollo-Jersey. Actas Iberoamericanas en Conservación Animal AICA, 8, 5-8. http://www.produccionanimal.com.ar/informacion_tecnica/raza_criolla/94-Trabajooo3.pdf

INAMHI (2013). Anuario Meteorológico 2013. Instituto Nacional de Meteorología e Hidrología. Quito-Ecuador. Disponible en: http://www.serviciometeorologico. gob.ec/docum_institucion/anuarios/meteorologicos/Am_2013.pdf

Juliano, N., Danelón, J. L., Fattore, R. O., Cantet, J. M., Martínez, R., Miccoli, F., Palladino, R. A. (2016). Crianza artificial de terneros de tambo utilizando sustitutos lácteos de distinto contenido energético. Revista de Investigaciones Agropecuarias, 42(1), 87-92. https://www.redalyc.org/articulo.oa?id=86445998016

Lagger, J. (2010). Crecimiento intensivo de cría y recría de vaquillonas, aplicando los principios de bienestar. Revista Veterinaria Argentina, 27(265), 1-28. http://www.produccion-animal.com.ar/produccion_bovina_de_leche/cria_ artificial/10-Crecimiento_Intensivo.pdf

Medina, S. M., Flores, F. A. I., Rivera, M. H. M., Álvarez, C. Y. M., López, R. R. (2017). Impacto económico del deterioro de los recursos naturales asociados con la eficiencia de la reproducción ganadera. Revista Mexicana de Agronegocios, 40, 649-650. https://www.redalyc.org/jatsRepo/141/14152127012/index.html

Moreno, S., Ibarra, F. A., Martín, M. H., Retes, R., Hernández, J. E., Jorge, E., Rodríguez, J. D. C. (2018). Importancia Económica De La Eficiencia De Producción De 
Becerros Utilizando Diversas Alternativas De Producción Y Manejo En El Centro De Sonora, México. Revista Mexicana de Agronegocios, 43, 107-116. https://ageconsearch.umn.edu/record/281302/files/10.-\%20Moreno\%20et\%20 al_becerros.pdf

Pavan, M. (2018). Guía de manejo sustentable y de buenas prácticas en la crianza artificial de terneras de tambo de o a 60 días. [Tesis Esp]. Argentina: Universidad Nacional de la Plata Facultad de Ciencias Veterinarias, (Consultado 01-07-2020). Disponible en: http://sedici.unlp.edu.ar/bitstream/handle/10915/68200/ Documento_completo.pdf?sequence $=1$

Pared, S. (2017). Consumo y crecimiento de terneros criados artificialmente con dietas solidas diferentes. (Tesis). (Argentina): Universidad Nacional del Centro de la Provincia de Buenos Aires, 27 p. (Consultado 02-07-2020), Disponible en: https://www.ridaa.unicen.edu.ar/xmlui/bitstream/handle/123456789/1377/ Pared,\%2oSergio\%2oIgnacio.pdf?sequence $=1$

Pineda, V., y Valiente, O. L. (2019). Efecto de la suplementación sobre el peso del ternero y la preñez y condición corporal de vacas del Bajo Chaco Paraguayo. Revista Veterinaria, 30(2), 46-51. https://revistas.unne.edu.ar/index.php/vet/ article/viewFile/4134/3771

Quintero, B. G. (2007). Sustitutos lecheros en la alimentación de terneros. REDVET. Revista electrónica de veterinaria, 8(5), 1-39. https://www.redalyc.org/ pdf/636/63612669002.pdf

Simeone, A., y Beretta, V. (2016). Destete precoz: una alternativa tecnológica eficiente y eficaz para los rodeos de cría en sistemas ganaderos pastoriles de América Latina. Cuban Journal of Agricultural Science, 50(1), 39-49. http://ref.scielo. org/6twq7f

Vazquez, Á. C. A., Puldón, R. Y., y Rodríguez, A. F. (2017). Efecto del empleo de raciones integrales en la conducta de terneros. Avances, 19(1), 51-65. https://pdfs. semanticscholar.org/b430/805a7b578d2f281c132eabf7195e1e4feo86.pdf

Ybalmea, R. (2015). Alimentación y manejo del ternero, objeto de investigación en el Instituto de Ciencia Animal. Cuban Journal of Agricultural Science, 49(2), 141-152. http://www.cjascience.com/index.php/CJAS/article/view/528 